\title{
Characterization of Deshi Jute (Corchorus capsularis) Germplasm Collected from Different Sources
}

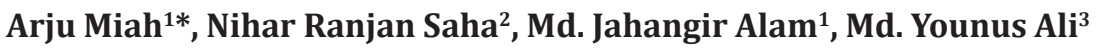 and Amit Kumar Basunia ${ }^{4}$}

${ }^{1}$ Genetic Resources and Seed Division, Bangladesh Jute Research Institute, Bangladesh

${ }^{2}$ Department of Biotechnology, Bangladesh Agricultural University, Bangladesh

${ }^{3}$ Fibre Quality Improvement Division, Bangladesh Jute Research Institute, Bangladesh

${ }^{4}$ Department of Horticulture, Bangladesh Agricultural University, Bangladesh

*Corresponding Author: Arju Miah, Genetic Resources and Seed Division, Bangladesh Jute

Research Institute, Bangladesh.

DOI: 10.31080/ASAG.2020.04.0870
Received: June 21, 2020

Published: June 30, 2020

(C) All rights are reserved by Arju Miah., et al.

\begin{abstract}
Forty accessions of white jute germplasm collected from different sources were characterized at JAES, Manikganj during kharif season in 2015. The accessions were characterized for twenty morpho-agronomic attributes as per Corchorus descriptor in order to select superior genotypes for the genetic improvement of jute. Considerable ranges of variability were observed in stem colour, petiole colour, stipule colour, plant technical height, base diameter, dry fiber weight and dry stick weight. Based on major yield contributing characters accessions 4869, 4621, 3394, 2199, 2839, 1478, 1777, 1923 and 2675 performed better in most of the cases than the control variety CVL-1 and CVE-3.
\end{abstract}

Keywords: Yield; Variability; Characterization; Corchorus; White Jute

\section{Introduction}

Bangladesh is a homeland of quality jute production and the second largest producer of jute around the world. Jute is the principal cash crop of Bangladesh. It occupies 5lh position after rice, pulses, oil seeds and wheat in respect of cultivated area [1], Bangladesh is not only the second largest producer of jute but she produces the best quality jute and leads the export market. Bangladesh produces 0.794 million tons of raw jute on about 0.392 million hectares of land with the national average production of 2.03 tons per hectare. It contributes about $6 \%$ of the total exporting earning and employs around 30 million people in different events such as cultivation, processing, carrying, marketing, research, trading and exporting jute [2]. Jute fibre is extracted from the bark of the jute plant and is known as a bast fibre. About 95 percent of the world's jute is produced in Bangladesh, India and Pakistan. Jute fibre is the cheapest fibre and is used in the manufacture of cordage, gunny cloth, gunny bags and other packaging materials for agricultural and industrial products. Now a days, novotex, blanket, fabrics, shopping bags, knilwear, nursery sheets and pots, micro crystal cellulose for pharmaceutical products, geo-jute and photo stable dye are being made from jute [3]. Jute constitutes an important source of employment and is of significant importance in the rural economy of Bangladesh.
Commercially jute is often referred to as the "golden fibre of Bangladesh", because of its immense contribution for the economy of this country. A good part of the total population of our country is engaged directly and indirectly in production and processing of jute. Jute exports constitute an important source of foreign exchange (12 - 13\%) earning in Bangladesh, During the year of 2012 - 2013, Bangladesh exported 619000 tons of raw jute and jute goods and earned about 16908 million taka [1].

Jute is a dicotyledonous plant of the genus Corchorus and family of the Tiliaccae. Jute is basically self-pollinated and has fourteen diploid chromosomes $(2 \mathrm{n}=14)$, The genus Corchorus contains about 50 - 60 species, which are distributed throughout the tropical regions of Africa, Australia, China and South-East Asia. Only two of the species, Corchorus capsularis L. and Corchorus olitorius L. are cultivated commercially for their fibre production. Corchorus capsularis L. has its center of origin in Indo-Burma [4] and C. olitorius L., however, originated in North Africa [5]. Corchorus capsularis L. is called deshi pat or white jute and its fibre is ordinarily whitish. While jute (C. capsularis L.) can grow both in low and high land and has better adaptability than the other cultivated jute species. Capsularis varieties in general, arc suitable for early sowing from March onwards, and perform better 
in low-lying areas. A moist heat is more favorable for the growth of the capsularis jute and nearly full-grown plants are tolerant to standing water. In general, capsularis shows flexibility in relation to drought and flood condition.

Although jute exhibits high socio-economic importance and its fibre is friendly to the environment for the producing countries, the global situation is confronted with number of problems. The prices paid to the farmers are not remunerative and subject to annual fluctuations. The area under jute in Bangladesh is declining and the crop is also being pushed more and more to the marginal lands. In our country, the number of recommended jute varieties is limited in terms of meeting the requirements of wide agroecological conditions. Most of these varieties are quite old and have narrow genetic base and susceptible to various biotic and abiotic stresses such as insect pests, diseases, drought, water logging, low temperatures and so on. All these factors combined with the increasing demand of jute in the world market, the new types of jute need to be developed to meet the various agro-industrial needs.

In order to increase the frequency of desired genotypes in breeding progenies, superior parents with high breeding values are needed. However, selection of such parents is a long term and tedious job. Genetic diversity is the fundamental law of plant breeding which is major tool and being used in parent selection for efficient hybridization programme. Modem breeding work needs diverse germplasm from which new' genes can be introduced into the existing cultivars in order to improve their yield, stability and resistance to pests and adverse conditions. The importance of genetic diversity in the improvement of a crop has been stressed in both self and cross-pollinated crop [6-8].

Therefore, the availability of transgressive segregant in any breeding programme depends upon the divergence of the involving parents, the quantification of genetic diversity' through biometrical procedures $[9,10]$ has made it possible to choose genetically diverse parents for a successful hybridization programme. Selection of parents based on geographic diversity alone is not always justified [11]. Moreover, evaluation of genetic diversity is important to know the source of genes for a particular trait within the available germplasm [12].

The Gene Bank of Bangladesh Jute Research Institute (BJRI) has been conserved about 6012 germplasm of jute, kenaf, mesta and allied fibre of both indigenous and exotic origin. Out of this 2400 germplasm are Corchorus capsularis. Already about 406 germplasm are characterized as per Corchorus Descriptor. The rest of germplasms need to characterize and find out superior germplasm for varietal development programme. Forty accessions of Deshi jute (Corchorus capsularis) germplasm received from different sources were characterized for morpho-agronomic traits to evaluate the promising types

\section{Methodology}

The experiment conducted by sowing seeds on 5 April, 2015. Forty entries along with the check varieties CVL-1 and CVE-3 were taken to this study. Each accession was sown in 5 rows of $3 \mathrm{~m}$ length; spacing was $30 \mathrm{~cm}$ between rows, 5-6 cm between plants and $1 \mathrm{~m}$ between plots. Standard cultural and inter-cultural practices were followed. Pigmentation data on stem colour, leaf colour, vein colour, petiole colour, stipule colour, bud colour, and fruit colour were collected at 60 days after sowing and pre-bud stage. Plants were harvested at 120 days after sowing and post harvest data were collected as per Corchorus Descriptor. Data were analyzed following standard statistical procedures. The significance of differences between the means of the treatments was evaluated by one way analysis of variance followed by Duncan's Multiple Range Test at the significance level of 5\% and least significance difference (LSD) test at $5 \%$ and $1 \%$ level of probability [13]. The statistical software Excel and MSTAT-C computer package program developed by Russel [14] were used for these analyses.

\section{Results}

Morphological characters of Corchorus capsularis germplasm

The plant technical height at harvest (120 days) ranged from 1.98 - 3.86m. The highest score was observed in accession no. 2839 (3.86 $\mathrm{m} /$ plant), followed by accessions 4869 (3.82 m/plant), variety CVL-1 (3.77 m/plant), acc. no. 3394 (3.62 m/plant), acc. no. 2199 (3.59 m/ plant) (Figure 1).

The mean performance of the major yield contributing characters and co-efficient of variation are presented in table 1 . Highest leaf angle $\left(55^{\circ}\right)$ was observed from accession number 637 while accession number 606 showed lowest leaf angle $\left(45^{\circ}\right)$. Largest leaf length $(16.86 \mathrm{~cm})$ was observed from accession number 628 while accession number 641 showed smallest leaf length $(10.67 \mathrm{~cm})$. Highest leaf width $(7.85 \mathrm{~cm})$ was observed from accession number 628 while accession number 641 showed lowest leaf width $(2.96$ $\mathrm{cm})$. Largest petiole length $(7.85 \mathrm{~cm})$ was observed from accession number 628 while accession number 641 showed smallest petiole length $(2.96 \mathrm{~cm})$. 


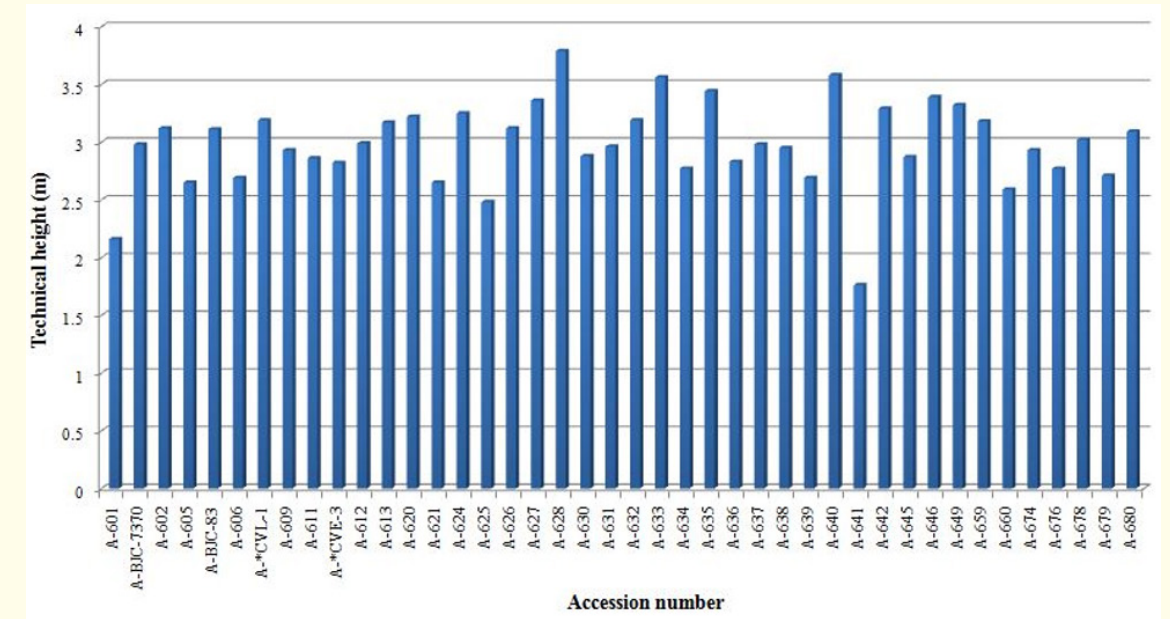

Figure 1: The plant technical height $(\mathrm{m})$ of different accessions of Desi jute at harvesting time.

\begin{tabular}{|c|c|c|c|c|c|c|c|c|c|}
\hline Acc. No. & $\begin{array}{c}\text { Leaf Angl } \\
\text { (dg) }\end{array}$ & $\begin{array}{l}\text { Leaf Lnth } \\
\text { (cm) }\end{array}$ & $\begin{array}{l}\text { Leaf Width } \\
\text { (cm) }\end{array}$ & $\begin{array}{l}\text { Petiol Lnth } \\
\text { (cm) }\end{array}$ & Node No. & $\begin{array}{c}\text { Base Dia } \\
(\mathrm{mm})\end{array}$ & $\begin{array}{l}\text { Middle Dia } \\
\text { (mm) }\end{array}$ & $\begin{array}{c}\text { Top Dia } \\
\text { (mm) }\end{array}$ & $\begin{array}{c}\text { Core Dia } \\
(\mathrm{mm})\end{array}$ \\
\hline 601 & 51 & 12.65 & 5.22 & 3.12 & 51 & 15.21 & 7.48 & 4.12 & 11.41 \\
\hline BJC - 7370 & 45 & 14.72 & 5.66 & 4.81 & 62 & 19.15 & 10.23 & 4.65 & 17.32 \\
\hline 602 & 52 & 15.17 & 6.23 & 5.12 & 65 & 17.29 & 12.44 & 5.25 & 18.58 \\
\hline 605 & 53 & 13.66 & 5.89 & 5.25 & 54 & 13.75 & 8.56 & 4.26 & 12.56 \\
\hline BJC - 83 & 46 & 14.58 & 6.22 & 6.11 & 69 & 23.25 & 12.33 & 5.36 & 18.25 \\
\hline 606 & 45 & 13.24 & 5.78 & 5.54 & 52 & 12.69 & 8.58 & 4.15 & 12.47 \\
\hline${ }^{*} \mathrm{CVL}-1$ & 48 & 14.65 & 5.93 & 6.22 & 68 & 23.58 & 12.45 & 5.28 & 18.36 \\
\hline 609 & 47 & 12.41 & 5.82 & 5.88 & 55 & 21.36 & 8.85 & 4.79 & 16.24 \\
\hline 611 & 50 & 11.88 & 5.11 & 5.15 & 54 & 20.58 & 9.58 & 4.96 & 16.87 \\
\hline${ }^{*} \mathrm{CVE}-3$ & 52 & 13.63 & 6.03 & 5.29 & 51 & 18.47 & 10.23 & 4.57 & 18.15 \\
\hline 612 & 51 & 14.11 & 6.78 & 6.08 & 56 & 22.56 & 12.14 & 5.04 & 17.26 \\
\hline 613 & 53 & 14.97 & 6.74 & 6.78 & 72 & 24.12 & 12.49 & 5.12 & 18.59 \\
\hline 620 & 46 & 14.22 & 6.77 & 6.85 & 73 & 24.51 & 12.58 & 5.23 & 18.62 \\
\hline 621 & 50 & 12.48 & 5.12 & 5.36 & 52 & 16.22 & 8.51 & 4.11 & 12.41 \\
\hline 624 & 55 & 15.49 & 7.22 & 6.92 & 71 & 24.67 & 12.78 & 5.54 & 18.56 \\
\hline 625 & 54 & 12.28 & 5.52 & 4.21 & 49 & 12.14 & 8.26 & 3.95 & 10.25 \\
\hline 626 & 46 & 14.52 & 6.59 & 6.65 & 69 & 23.98 & 12.15 & 5.25 & 17.98 \\
\hline 627 & 50 & 15.77 & 7.14 & 6.92 & 72 & 24.77 & 12.27 & 5.28 & 18.57 \\
\hline 628 & 51 & 16.86 & 7.97 & 7.85 & 78 & 25.13 & 13.23 & 6.77 & 20.54 \\
\hline 630 & 49 & 13.23 & 6.96 & 5.99 & 61 & 19.36 & 10.29 & 4.87 & 13.27 \\
\hline 631 & 53 & 14.11 & 6.85 & 6.07 & 59 & 20.58 & 10.58 & 4.12 & 16.59 \\
\hline 632 & 53 & 14.97 & 6.81 & 6.84 & 68 & 24.45 & 12.48 & 5.29 & 18.48 \\
\hline 633 & 47 & 16.65 & 7.78 & 7.61 & 76 & 24.98 & 13.06 & 6.35 & 20.09 \\
\hline 634 & 52 & 11.66 & 4.98 & 4.62 & 55 & 21.56 & 9.88 & 4.58 & 12.95 \\
\hline 635 & 46 & 15.89 & 7.47 & 7.24 & 74 & 24.75 & 12.86 & 6.08 & 19.83 \\
\hline 636 & 48 & 13.76 & 6.25 & 5.27 & 51 & 21.59 & 10.25 & 4.89 & 14.65 \\
\hline 637 & 55 & 13.62 & 5.97 & 3.79 & 61 & 23.55 & 11.65 & 5.21 & 15.28 \\
\hline
\end{tabular}




\begin{tabular}{|c|c|c|c|c|c|c|c|c|c|}
\hline 638 & 54 & 14.14 & 6.58 & 5.25 & 60 & 16.25 & 10.47 & 5.22 & 16.82 \\
\hline 639 & 52 & 13.92 & 5.87 & 5.27 & 58 & 14.15 & 9.58 & 4.25 & 15.73 \\
\hline 640 & 46 & 16.55 & 7.25 & 7.49 & 75 & 25.04 & 13.11 & 6.52 & 20.25 \\
\hline 641 & 48 & 10.67 & 4.55 & 2.96 & 46 & 11.24 & 6.45 & 3.14 & 9.76 \\
\hline 642 & 49 & 15.93 & 7.14 & 7.27 & 71 & 23.56 & 12.86 & 6.23 & 18.74 \\
\hline 645 & 52 & 13.87 & 6.59 & 5.83 & 58 & 16.54 & 11.25 & 4.54 & 16.51 \\
\hline 646 & 54 & 15.98 & 7.33 & 7.18 & 72 & 24.29 & 12.45 & 6.55 & 19.89 \\
\hline 649 & 48 & 15.46 & 7.09 & 7.08 & 71 & 23.96 & 12.14 & 6.11 & 19.24 \\
\hline 659 & 53 & 15.23 & 7.12 & 7.02 & 69 & 23.55 & 12.23 & 5.98 & 18.92 \\
\hline 660 & 55 & 12.87 & 5.88 & 5.21 & 53 & 12.44 & 8.56 & 4.56 & 15.53 \\
\hline 674 & 54 & 13.21 & 6.21 & 5.32 & 56 & 21.87 & 11.25 & 4.77 & 17.54 \\
\hline 676 & 49 & 13.45 & 6.23 & 5.19 & 55 & 20.45 & 10.21 & 4.58 & 14.86 \\
\hline 678 & 47 & 14.87 & 6.78 & 6.93 & 68 & 23.56 & 12.23 & 4.25 & 18.52 \\
\hline 679 & 50 & 12.99 & 6.22 & 5.81 & 51 & 14.25 & 10.51 & 4.73 & 14.15 \\
\hline 680 & 53 & 15.05 & 7.02 & 6.75 & 66 & 22.59 & 11.99 & 4.42 & 18.58 \\
\hline Range & $45-55$ & $10.67-$ & $4.55-7.97$ & $2.96-7.85$ & $46-78$ & $11.24-$ & $6.45-13.23$ & $3.14-6.77$ & $9.76-20.54$ \\
\hline Mean & 45 & 14.85 & 6.19 & 6.18 & 63.24 & 19.68 & 10.44 & 4.44 & 16.03 \\
\hline CV (\%) & 6.79 & 6.28 & 13.56 & 17.58 & 14.29 & 15.67 & 12.48 & 15.85 & 16.72 \\
\hline
\end{tabular}

Table 1: Range, mean and co-efficient of variation (CV \%) of twelve characters of forty Corchorus capsularis germplasm.

*= Check Variety.

Highest node number (78) was observed from accession number 628 while accession number 641 showed lowest node number (46). Largest base diameter (25.13 mm) was observed from accession number 628 while accession number 641 showed smallest base diameter $(11.24 \mathrm{~mm})$. Largest middle diameter (13.23 mm) was observed from accession number 628 while accession number 641 showed smallest middle diameter (6.45 $\mathrm{mm})$. Largest top diameter $(6.77 \mathrm{~mm})$ was observed from accession number 628 while accession number 641 showed smallest top diameter (3.14 mm). Largest core diameter (20.54 $\mathrm{mm}$ ) was observed from accession number 628 while accession number 641 showed smallest core diameter (9.76 mm) (Table 1).

Dry fibre weight ranged from 8.29 - $20.48 \mathrm{~g} /$ plant. The highest dry fibre weight was observed in accession no. 4869 (20.48 g/plant) and followed by accessions 4621 (19.49 g/plant), accession no. 1478 (18.94 g/plant), variety CVL-1 (15.69 g/plant) (Figure 2). Dry stick weight ranged from 15.48 - $55.56 \mathrm{~g} /$ plant. The highest score was recorded in accession 4621 (55.56 g/plant) followed by acc. no. 4869 (50.95 g/plant), acc. no.1478 (49.58 g/plant), variety CVL-1 (15.69 g/plant) and acc. no. 2839 (43.84 g/plant) (Figure 3).
Qualitative characters and growth habits of Corchorus capsularis germplasm

Pigmentation data on stem colour, leaf colour, vein colour, petiole colour, stipule colour, bud colour, and fruit colour are presented in table 2.

Red stem color was found in accession number 1478, 2699. Stem color was green in accession number 1533, 1777, 1799, 1910, 192, 2019, 2097, 2114, 2120, 2199, 2274, 2349, 2417, 2556, 2675, CVL-1, 2764, 2814, 2797, 2814, 2839, 2861, 2967, 3145, 3258, 3394, 3551, 3618, 3857, 3935, 4621, 4724, 4869, 5927, CVE-3 while either red or green colored stems were shown by accession number 1879, 2395, 2797, 2895, 3309, 3449 (Table 2).

Leaf color was green in all accession. Vein color was green in accession number 1533, 1777, 1799, 1910, 1923, 2019, 2097, 2114, 2120, 2199, 2274, 2349, 2395, 2417, 2556, 2675, *CVL-1, 2764, $2797,2814,2839,2861,2895,2967,3145,3258,3394,3449,3551$, $3618,3857,3935,4621,4724,4869,5927,{ }^{*}$ CVE-3. Vein color was reddish-green in accession number 1478, 1879, 2699, 3193, 3309. All of the accessions were stipulated (Table 2). 


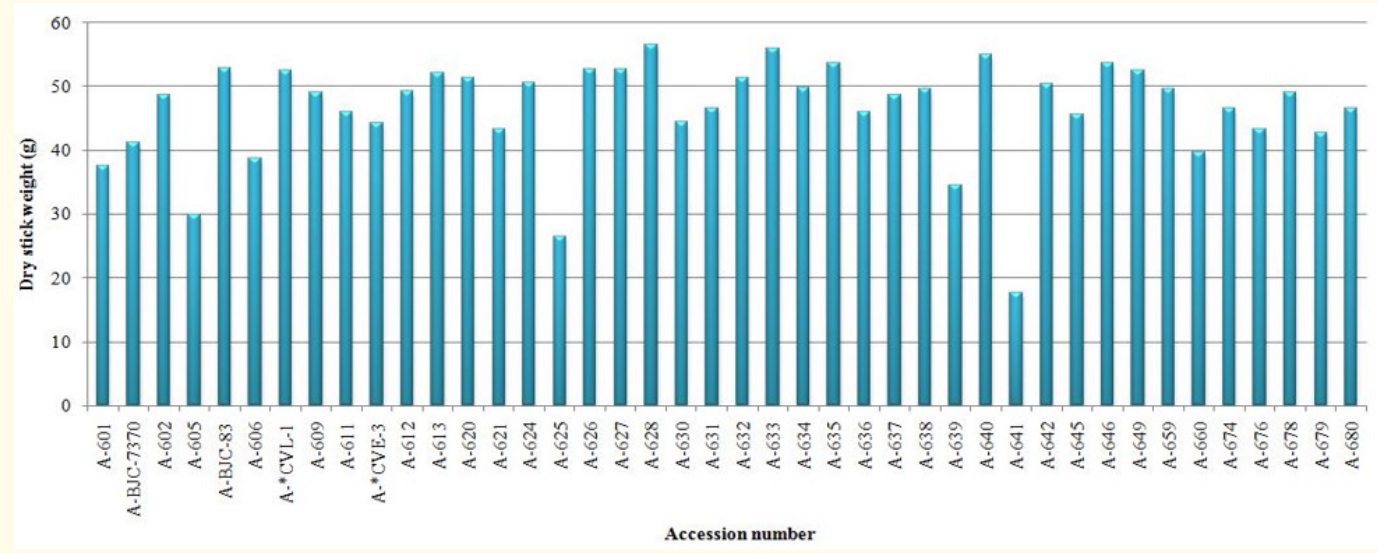

Figure 2: Dry fibre weight (g/plant) of different accessions of Desi jute.

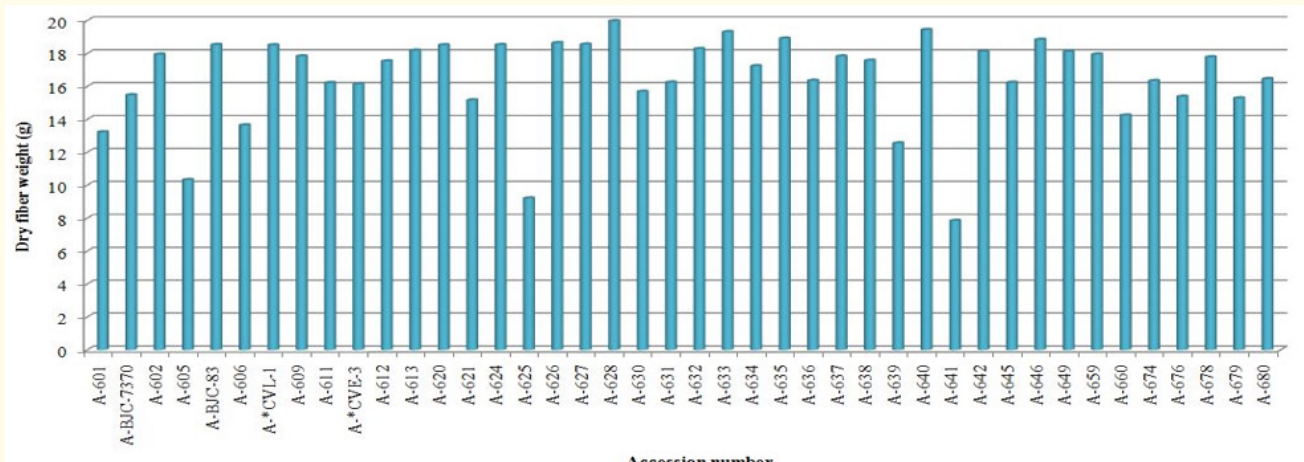

Figure 3: Dry stick weight (g/plant) of different accessions of Desi jute.

\begin{tabular}{|c|c|c|c|c|c|c|c|c|c|c|}
\hline Acc. No. & $\begin{array}{l}\text { Stem } \\
\text { color }\end{array}$ & $\begin{array}{l}\text { Leaf } \\
\text { color }\end{array}$ & Vein Color & $\begin{array}{l}\text { Petiole } \\
\text { color }\end{array}$ & Stipule & Stipule color & Bud color & Fruit Color & Branch habit & Leaf shape \\
\hline 1478 & $\mathrm{R}$ & $G$ & $\mathrm{G} / \mathrm{R}$ & $\mathrm{RG}$ & + & G & $\mathrm{G}$ & G & Rudimentary & Ovate \\
\hline 1533 & $\mathrm{G}$ & $\mathrm{G}$ & $\mathrm{G}$ & GR & + & $\mathrm{G}$ & $\mathrm{G}$ & $\mathrm{G}$ & Non branch & Ovate \\
\hline 1777 & $\mathrm{G}$ & $\mathrm{G}$ & G & GR & + & $G$ & $\mathrm{G}$ & G & Non branch & Ovate \\
\hline 1799 & $\mathrm{G}$ & $\mathrm{G}$ & $G$ & $G$ & + & $\mathrm{G}$ & $\mathrm{G}$ & G & Rudimentary & Ovate-lanceolate \\
\hline 1879 & $\mathrm{G} / \mathrm{R}$ & G & GR & $\mathrm{R}$ & + & GR & G & G & Non branch & Ovate-lanceolate \\
\hline 1910 & $\mathrm{G}$ & $\mathrm{G}$ & $G$ & GR & + & $\mathrm{G}$ & $\mathrm{G}$ & $\mathrm{G}$ & Sparse & Ovate-lanceolate \\
\hline 1923 & $\mathrm{G}$ & $\mathrm{G}$ & $\mathrm{G}$ & GR & + & $\mathrm{G}$ & $\mathrm{G}$ & G & Non branch & Ovate-lanceolate \\
\hline 2019 & $\mathrm{G}$ & $\mathrm{G}$ & G & GR & + & $\mathrm{G}$ & $\mathrm{G}$ & G & Rudimentary & Ovate-lanceolate \\
\hline 2097 & $\mathrm{G}$ & $\mathrm{G}$ & $\mathrm{G}$ & GR & + & $\mathrm{G}$ & $\mathrm{G}$ & G & Non branch & Ovate-lanceolate \\
\hline 2114 & G & G & G & GR & + & G & G & $\mathrm{G}$ & Non branch & Ovate-lanceolate \\
\hline 2120 & $\mathrm{G}$ & $\mathrm{G}$ & $\mathrm{G}$ & G & + & $\mathrm{G}$ & $\mathrm{G}$ & LR & Non branch & Ovate-lanceolate \\
\hline 2199 & G & G & $\mathrm{G}$ & $\mathrm{G}$ & + & G & $\mathrm{G}$ & $G$ & Rudimentary & Ovate-lanceolate \\
\hline 2274 & $\mathrm{G}$ & $\mathrm{G}$ & $\mathrm{G}$ & $\mathrm{G} / \mathrm{R}$ & + & $\mathrm{G}$ & $\mathrm{G}$ & $\mathrm{G}$ & Non branch & Ovate-lanceolate \\
\hline 2349 & G & $\mathrm{G}$ & $G$ & GR & + & G & G & $G$ & Rudimentary & Ovate-lanceolate \\
\hline
\end{tabular}




\begin{tabular}{|c|c|c|c|c|c|c|c|c|c|c|}
\hline 2395 & $\mathrm{G} / \mathrm{R}$ & G & G & G & + & G & G & G & Non branch & Ovate-lanceolate \\
\hline 2417 & $G$ & G & G & G & + & G & G & G & Rudimentary & Ovate-lanceolate \\
\hline 2556 & G & G & G & G & + & G & G & G & Non branch & Ovate-lanceolate \\
\hline 2675 & G & G & G & G & + & $G$ & G & $\mathrm{G}$ & Non branch & Ovate-lanceolate \\
\hline 2699 & $\mathrm{R}$ & G & $\mathrm{G} / \mathrm{R}$ & G & + & $\mathrm{G} / \mathrm{R}$ & G & G & Non branch & Ovate-lanceolate \\
\hline *CVL-1 & G & G & $G$ & G & + & $G$ & G & G & Non branch & Ovate-lanceolate \\
\hline 2764 & $G$ & G & G & $G$ & + & G & G & $\mathrm{G}$ & Non branch & Ovate-lanceolate \\
\hline 2797 & $\mathrm{G} / \mathrm{R}$ & G & G & $\mathrm{G} / \mathrm{R}$ & + & G & G & G & Non branch & Ovate \\
\hline 2814 & $G$ & G & G & G & + & $G$ & G & G & Non branch & Ovate-lanceolate \\
\hline 2839 & G & G & G & $\mathrm{G}$ & + & G & G & G & Non branch & Ovate-lanceolate \\
\hline 2861 & $\mathrm{G}$ & G & G & G & + & G & G & G & Non branch & Ovate-lanceolate \\
\hline 2895 & $\mathrm{G} / \mathrm{R}$ & G & G & G & + & G & G & G & Non branch & Ovate-lanceolate \\
\hline 2967 & $G$ & G & $\mathrm{G}$ & $\mathrm{G} / \mathrm{R}$ & + & G & G & G & Non branch & Ovate \\
\hline 3145 & G & G & G & $\mathrm{G} / \mathrm{R}$ & + & G & G & G & Non branch & Ovate \\
\hline 3193 & $\mathrm{R}$ & G & $\mathrm{G} / \mathrm{R}$ & $\mathrm{G} / \mathrm{R}$ & + & $\mathrm{G} / \mathrm{R}$ & G & G & Non branch & Ovate-lanceolate \\
\hline 3258 & G & G & $\mathrm{G}$ & $G$ & + & G & G & G & Non branch & Ovate-lanceolate \\
\hline 3309 & $\mathrm{G} / \mathrm{R}$ & G & $\mathrm{G} / \mathrm{R}$ & $\mathrm{G} / \mathrm{R}$ & + & $\mathrm{G} / \mathrm{R}$ & G & G & Non branch & Ovate-lanceolate \\
\hline 3394 & $G$ & G & $\mathrm{G}$ & $\mathrm{G} / \mathrm{R}$ & + & $G$ & G & G & Non branch & Ovate-lanceolate \\
\hline 3449 & $\mathrm{G} / \mathrm{R}$ & G & G & $\mathrm{G} / \mathrm{R}$ & + & G & G & G & Non branch & Ovate-lanceolate \\
\hline 3551 & $\mathrm{G}$ & G & G & $G$ & + & G & G & G & Non branch & Ovate-lanceolate \\
\hline 3618 & G & G & G & G & + & G & G & G & Non branch & Ovate-lanceolate \\
\hline 3857 & G & G & G & $\mathrm{G} / \mathrm{R}$ & + & $\mathrm{R}$ & G & G & Non branch & Ovate-lanceolate \\
\hline 3935 & G & G & G & G & + & G & G & G & Non branch & Ovate-lanceolate \\
\hline 4621 & G & G & G & G & + & G & G & G & Non branch & Ovate \\
\hline 4724 & G & G & G & $\mathrm{G} / \mathrm{R}$ & + & G & G & G & Non branch & Ovate-lanceolate \\
\hline 4869 & G & G & G & $\mathrm{G} / \mathrm{R}$ & + & G & G & G & Non branch & Ovate-lanceolate \\
\hline 5927 & G & G & G & $G$ & + & G & G & G & Non branch & Ovate-lanceolate \\
\hline${ }^{*}$ CVE-3 & G & G & G & G & + & G & G & G & Non branch & Ovate-lanceolate \\
\hline
\end{tabular}

Table 2: Pigmentation of Corchorus capsularis germplasm along with check variety CVL-1 and CVE-3.

G: Green; R: Red; LR: Light Red; “+”: Present; * Check variety.

Stipule color was green in accession number 1478, 1533, 1777, 1799, 1910, 1923, 2019, 2097, 2114, 2120, 2199, 2274, 2349, 2395, 2417, 2556, 2675, CVL-1, 2764, 2797, 2814, 2839, 2861, 2895, 2967, 3145, 3258, 3394, 3449, 3551, 3618, 3935, 4621, 4724, 4869, 5927 and CVE-3. Stipule color was red in accession number 3857. Stipule color was reddish-green in accession number 1879, 2699, 3193 and3309. Bud color was green in all accessions. Fruit color was light red in accession 2120. All of the rest accessions were green (Table 2).

Rudimentary branching habit was found in accession number 1478, 1799, 2019, 2199, 2349 and 2417. Rest of the accessions was showing no branching. Leaf shape was ovate in accessions number 1478, 1533, 1777, 2797, 2967, 3145 and 4621. Rest of the accessions showed ovate to lanceolate shaped leaf (Table 2).

\section{Discussion}

Mean sum of square for plant height was highly significant (Table 1). The highest score of plant height was recorded in accession 2839 (3.86 m/plant) which was followed by accessions 4869 (3.82 m/plant), variety CVL-1 (3.77 m/plant), acc. no. 3394 (3.62 m/plant), acc. no. 2199 (3.59 m/ plant) (Figure 1). High heritability was found for the plant height was reported by Dutta et al. (1973). Variation of plant height among different genotypes of Kenaf was shown on figure 1 . The highly significant MS value indicates existence of considerable difference for this trait. Coefficient of variance for plant 
height was $6.79 \%$ which clearly indicates the significant difference among the accessions. A narrow range of difference between the phenotypic coefficient of variation (PCV) and genotypic coefficient of variation (GCV) indicating less environmental influence on the phenotypic expression of plant height and it is mostly governed by genetic factors. Hence, selection will be effective on phenotypic basis. High heritability coupled with moderate genetic advance for plant height indicating the predominance of additive gene effects on plant height. So we have scope to improve this trait before using it in breeding program.

Mean sum of square for base diameter was highly significant (Table 1) indicating existence of considerable difference among the genotypes for this trait. Base diameter was ranged from 11.24 - $25.13 \mathrm{~mm}$. Largest base diameter (25.13 mm) was observed from accession number 628 while accession number 641 showed smallest base diameter (11.24 mm). Mostofa., et al. [15] also reported the highest heritability for base diameter in Tossa Jute. The genotypes having the highest and the lowest base diameter were depicted in table 1. $15.67 \%$ of coefficient of variance was observed for base diameter which indicates the significant difference among the kenaf accessions. Here, selection will be effective on the basis of phenotype. High heritability coupled with high genetic advance for base diameter indicates that the selection of such character may be useful for improvement of the crops.

The analysis of variance revealed highly significant differences among the genotypes with respect to number of nodes per plant. Number of nodes ranged from 46 - 78. Highest node number (78) was observed from accession number 628 while accession number 641 showed lowest node number (46). Narrow difference between GCV and PCV for this trait indicated the less environmental influence. Mean node number was 63.24 with $14.29 \%$ coefficient of variance (Table 1). Similar findings were reported by Ghosdastidar., et al. [16] and Johnson., et al [17]. This value notifies that the character is governed by additive genes and selection will be rewarding for improvement of such trait.

Petiole length ranged from $2.96-7.85 \mathrm{~cm}$. Highly significant differences among the genotypes were observed for petiole length. Largest petiole length $(7.85 \mathrm{~cm})$ was observed from accession number 628 while accession number 641 showed smallest petiole length $(2.96 \mathrm{~cm})$. Mean petiole length was $6.19 \mathrm{~cm}$ with $17.58 \%$ coefficient of variance (Table 1). So, selection is ineffective without improvement of this character. Chaudhury., et al. [18] observed the same findings in Tossa Jute.
Dry fibre weight ranged from 7.85 - 19.98 gm/plant (Figure 2). The highest dry fibre weight was observed in accession no. 4869 (20.48 g/plant) and followed by accessions 4621 (19.49 g/plant), accession no. 1478 (18.94 g/plant), variety CVL-1(15.69 g/plant) (Figure 2). Mean fibre weight was $12.09 \mathrm{gm}$ with the coefficient of variance $21.39 \%$. Hence, selection will be effective on phenotypic basis. This findings was also supported by Sasmol and Chakrabarty [19] and Dutta., et al. [20] in Kenaf.

A wide variation was found among the genotypes for the stick weight or core weight. It varied 17.59 - 56.48 gm significantly among the genotypes with an overall mean of $33.29 \mathrm{gm}$. The entry 641 showed the lowest stick weight and the highest stick weight was recorded by the entry 628 (Figure 3). The coefficient of variance for stick weight was $22.65 \%$. Manjunatha and sheriff [21] recorded similar findings in Kenaf. This character is highly efficient for selection.

\section{Conclusion}

Among the accessions characterized, nine accessions viz. 4869, 4621, 3394, 2199, 2839, 1478, 1777, 1923 and 2675 performed better in respect of major yield contributing characters than the controls CVL-1 and CVE-3. Therefore, these nine accessions may be selected for further study.

\section{Bibliography}

1. BBS. Statistical Pocket Book of Bangladesh. Bangladesh Bureau of Statistics. Planning Division, Ministry of Planning. Govt. Peoples Republic of Bangladesh (2013).

2. BBS. Year Book of Agricultural Statistics of Bangladesh. Bangladesh Bureau of Statistics. Statistics Division, Ministry of Planning, Government of the People's Republic of Bangladesh, Dhaka, Bangladesh (2012): 37.

3. Islam MR. Genetic divergence in Corcharous alitorbus L. MS Thesis submitted to Institute of Postgraduate Studies in Agriculture (IPSA), Salna, Gazipur (1996).

4. Singh KB., et al. "Genetic divergence for yield and its components in greengram". Mysore Journal of Agricultural Sciences 10 (1976): 535-544.

5. Kundu BC., et al. "Jute in India”. Indian central Jute Committee, Kalkata (1959) 10. 
6. Griffin B and Lindstorm EW. "A study of combining abilities of corn inbreeds having varying proportions of com belt and non-com belt germplasm". Agronomy Journal 46 (1954): 545552.

7. Murty BR., et al. "Combining ability and genetic diversity in some varieties in Linum usitatissium". Indian Journal of Genetics 26 (1966): 21-36.

8. Gaur PC., et al. "Studies on genetic divergence in potato". Euphytica 27 (1978): 361-368.

9. Anderson. E. "A semigraphical method for the analysis of complex problems". Proceedings of the National Academy of Sciences 48 (1957): 923-927.

10. Rao CR. "Advance Statistical Methods in Biometrical Research". John wiley and sons. New York (1952).

11. Sherestha VS. "Genetic variability correlation and path analysis studies in jute". MS thesis submitted to Rajendra Agricultural University, Bihar, Pusa, India (1991): 96-98.

12. Tomooka N. "Genetic diversity and landrace differentiation of mungbean, Vigna radiata (L) wilczek. and evaluation of its wild relatives (The. subgenus Ceratolropics) as breeding materials". Tech. Bul Trop. Res. Center, Japan. No, 28, Ministry of Agriculture, Forestry and Fisheries, Japan (1991): 1.

13. Gomez KA and Gomez AA. Statistical procedures for agricultural research (2 ed.). (1984): 188-240.

14. Russel DF. MSTAT-C Package programme. Crop and Soil Science Department, Michigan State University, USA (1986).

15. Mostofa MG., et al. "Genetic variability, heritability and correlation studies in Kenaf (Hibiscus Cannabinus L.)". Online Journal of Biological Sciences 2.6 (2002): 422-424.

16. Ghosdastidar KK and Das PK. "Selection breeding in Tossa Jute (Corchorus olitorius L.)”. Perspective CytoGenetics 4 (1984): 563-567.

17. Johnson HW., et al. "Estimates of genetic and environmental variability in soybean”. Agronomy Journal 47 (1955): 314-318.
18. Chaudhury SK. Studies on the genetic variability in Jute and its utilization for higher yield. Bangladesh Agricultural Research Institute, Annual Report 1984. JB 7 (1984): 11.

19. Sasmol BC and Chakroborty K. "Correlation and path coefficient analysis of yield component in Mesta". Indian Journal of Heredity 10 (2004): 19-27.

20. Dutta AN., et al. "Heritability and correlation studies in Kenaf". Indian Journal of Genetics and Plant Breeding 55.3 (1973): 279-282.

21. Manjunatha S and Sheriff RA. "Variability, correlation and divergence studies in Kenaf". 2 (1991): 569.

\section{Assets from publication with us}

- Prompt Acknowledgement after receiving the article

- Thorough Double blinded peer review

- Rapid Publication

- Issue of Publication Certificate

- High visibility of your Published work

Website: https://www.actascientific.com/

Submit Article: https://www.actascientific.com/submission.php Email us: editor@actascientific.com

Contact us: +919182824667 